\title{
Dust properties in NGC 6611
}

\author{
A.M. Orsatti ${ }^{1,2,4,5}$, E.I. Vega ${ }^{1,2,3,4,5}$, and H.G. Marraco ${ }^{1,4}$ \\ 1 Facultad de Ciencias Astronómicas y Geofísicas, Universidad Nacional de La Plata, Paseo del Bosque s/n, 1900, La Plata, \\ Argentina \\ 2 Programa de Fotometría y Estructura Galáctica, PROFOEG, CONICET, Paseo del Bosque s/n, 1900, La Plata, Argentina \\ 3 Instituto de Astronomía y Física del Espacio, C. C. 67 Suc. 28, 1428, Buenos Aires, Argentina \\ 4 Member of the Carrera del Investigador Científico of CONICET \\ 5 Visiting Astronomer, Complejo Astronómico El Leoncito, operated under agreement between the Consejo Nacional de \\ Investigaciones Científicas y Técnicas de la República Argentina and the Universities of La Plata, Córdoba, and San Juan
}

Received July 28, 1999; accepted February 25, 2000

\begin{abstract}
An investigation on dust properties in the NW portion of NGC 6611 has been conducted polarimetrically to test the existence of abnormal extinction in that section of the open cluster in an independent way.

As previously suggested for the $\eta$ Carinae nebula by Tapia et al. (1988b) and subsequently confirmed by Marraco et al. (1993), the canonical relation between $E_{V-K} / E_{B-V}$ and $\lambda_{\max }$ (the wavelength of maximun interstellar polarization) is not valid for stars belonging to dusty $\mathrm{H}$ II regions, as is the case for the observed portion of M 16. This may arise mainly from the presence of silicate grains of a slightly larger size than the standard ISM and also from a considerable increase in mean graphite grain size, according to previous results from Chini \& Wargau (1990).

About $50 \%$ of the observed stars in NGC 6611 present indications of intrinsic polarization in their measurements; a similar percentage was found in IC 2944 (Vega et al. 1994), another young open cluster in close relation with an $\mathrm{H}$ II region.
\end{abstract}

Key words: ISM: dust, extinction - Galaxy: open clusters and associations: individual (NGC 6611) - ISM: HII regions - ISM: magnetic fields

\section{Introduction}

NGC 6611 (C1816-138) is a stellar rich open cluster located in the Sagittarius spiral arm, in the plane of the

Send offprint requests to: A.M. Orsatti, e-mail: aorsatti@fcaglp.fcaglp.unlp.edu.ar

* Table 1 only available in electronic form at CDS via anonymous ftp to cdsarc.u-strasbg.fr (130.79.128.5) or via http://cdsweb.u-strasbg.fr/Abstract.html
Galaxy $\left(l=17^{\circ} 0, b=0.8\right)$. It is embedded in an ionized hydrogen complex (M 16), an extensive nebulosity connected with dense dust clouds. The central portion of the cluster is part of the North-West of M 16, but nowadays it is assumed that it is spread all over the complex (e.g. de Winter et al. 1997).

Prior to the era of the infrared observational campaigns, the extinction law in NGC 6611 was considered to be fairly normal. Gebel (1968) obtained a value of $R_{V}=2.5 \pm 0.6$ from a comparison of the radio emission measure for the $\mathrm{H}$ II region with $\mathrm{H} \alpha$ and $\mathrm{H} \beta$ fluxes. Johnson (1968) obtained a mean value of $R_{V}=2.9 \pm 0.5$ for NGC 6611 from an average of estimates from the variable-extinction technique $\left(R_{V}=3.4 \pm 0.7\right)$ and the cluster diameter method $\left(R_{V}=2.3\right)$. The value obtained by Turner (1994) from the variable-extinction method was $R_{V}=2.99 \pm 0.08$, with only a localized region on the dusty north side of the cluster displaying evidence for a larger value of 4.42 .

Many later investigations on NGC 6611, mostly based on IR studies (Sagar \& Joshi 1979; Chini \& Krugel 1983; Hillenbrand et al. 1993; de Winter et al. 1997; and others) concluded that the extinction in the NW area is caused either by intracluster or by circumstellar dust while showing variable degrees of anomaly. This in turn causes uncertainties in the determination of the distance to the cluster and consequently has some influence on the conclusions regarding the evolution of NGC 6611 itself. Also, it is generally accepted that the matter in front of the cluster is responsible for an excess $E_{B-V}=0.50 \mathrm{mag}$ (e.g. Sagar \& Joshi 1979; Thé et al. 1990; Hillenbrand et al. 1993) and that a normal extinction law, characterized by $R_{V}=3.1 \pm 0.1$, is valid for the foreground dust.

Our intention is to test independently the existence of abnormal extinction in NGC 6611 through the use of polarimetric techniques. Via polarimetry, it is possible to 
detect stars suspected of having circumstellar envelopes and to remove them from the rest; and then, to concentrate on the extinction caused by the intra-cluster dust.

\section{Observations}

The stars to be observed polarimetrically were selected from the work of Walker (1961) covering the central core and some dusty regions north of it, where Turner (1974) detected about a dozen stars suffering of an abnormal extinction law.

Observations in the $U B V R_{\mathrm{KC}} I_{\mathrm{KC}}$ bands (KC: KronCousins, $\lambda_{U_{\text {eff }}}=0.36 \mu \mathrm{m}, F W H M=0.05 \mu \mathrm{m} ; \lambda_{B_{\text {eff }}}$ $=0.44 \mu \mathrm{m}, F W H M=0.06 \mu \mathrm{m} ; \lambda_{V_{\text {eff }}}=0.53 \mu \mathrm{m}$, $F W H M=0.06 \mu \mathrm{m} ; \lambda_{R_{\text {eff }}}=0.69 \mu \mathrm{m}, F W H M=$ $\left.0.18 \mu \mathrm{m} ; \lambda_{I_{\mathrm{eff}}}=0.83 \mu \mathrm{m}, F W H M=0.15 \mu \mathrm{m}\right)$ were carried out using the five-channel photopolarimeter of the Torino Astronomical Observatory, attached to the $2.15 \mathrm{~m}$ telescope at the Complejo Astronómico El Leoncito (San Juan, Argentina). They were performed on 4 nights (August 26/29) in 1995 and 2 nights (June 18/19) in 1996, both runs of suitable quality. Standard stars for null polarization and for the zero point of the polarization position angle were taken from Clocchiatti \& Marraco (1988).

Table 1 lists on its left side, for the 39 observed stars, the percentage polarization $\left(P_{\lambda}\right)$, the position angle of the electric vector $\left(\theta_{\lambda}\right)$ in the equatorial coordinate system and their respective mean errors for each filter. We indicate also the number of independent integrations with each filter. Star identifications are taken from the work of Walker (1961).

\section{Results}

Assuming that the polarization is of interstellar origin, the wavelength at which maximum polarization $P_{\max }$ occurs can be computed by observing in several photometric bandpasses. This wavelength $\lambda_{\max }$ is a function of the optical properties and characteristic particle size distribution of the aligned grains (McMillan 1978; Wilking et al. 1980). The maximum polarization (in microns) at which $P_{\max }$ (in percentage) occurs has been calculated by fitting the observed interstellar polarization in the $U B V R I$ bandpasses to the standard Serkowski polarization law (Serkowski 1973):

$P_{\lambda} / P_{\max }=\exp \left[-K \ln ^{2}\left(\lambda_{\max } / \lambda\right)\right]$

and adopting $K=1.15$.

The left side of Table 2 lists the $P_{\max }$ and $\lambda_{\max }$ values for 32 stars, excluding those from Table 1 with errors in their $P_{V}$ values higher than $15 \%$ and $P_{B}$ higher than $20 \%$. If the polarization is well represented by the Serkowski relation, $\sigma_{1}$ (the unit weight error of the fit) should not be higher than 1.5 because of the weighting scheme; a higher value could indicate the presence of intrinsic polarization.

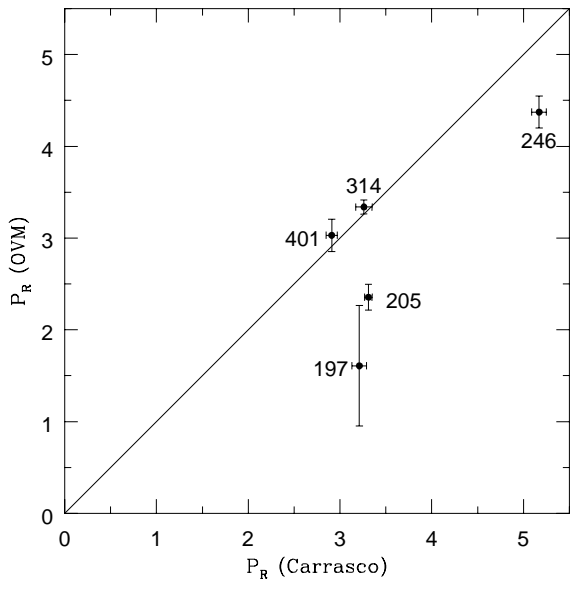

Fig. 1. Relation between $P_{R}$ polarizations values for stars observed in this work (OVM) and by Carrasco et al. (1975)

The mathematical expression used to determine the individual $\sigma_{1}$ values can be found as a footnote. Excesses are taken from the works of Hillenbrand et al. (1993), Thé et al. (1990), de Winter et al. (1997) or they have been calculated according to Feinstein \& Marraco (1971), using $U B V$ colors from Hillenbrand et al. (1993). Star No. 313 shows indications of photometric variability, as it results from a comparison between magnitudes and colors from Thé et al. (1990) and from Chini \& Wargau (1990): 13.29 $(V), 0.36(B-V)$ and $-0.14(U-B)$ versus $12.95,0.58$ and -0.19 , respectively.

Following Marraco et al. (1993), we have intended to remove the effects on the measured polarizations of the interstellar dust located in front of the complex, leaving only the intra-cluster variations of the extinction. To do so, we adopted a more conservative approach than in Marraco et al. (1993) or in Vega et al. (1994), since in the present case the foreground absorption over the region under study is somewhat patchy and that particular situation prevents us from performing a fair modeling of the effects of the foreground dust parameters in terms of latitude and longitude.

We selected from the observed stars those which seem to be the least affected by reddening and having low values in their polarizations: stars Nos. 349, 367, 374 and 455, excluding star No. 411 by reasons discussed later. Please note that "frontside" is a concept not directly related to the conditions of "foreground" and "member". The ideal frontside star is one that has all foreground dust in front of it and all intracluster dust behind it. Sometimes this condition is met by the member stars just in front of most of intracluster dust as explained in Marraco et al. (1993). Those four already mentioned stars, which are hereinafter referred to as "frontside" stars, are noted with asterisks in Tables 1 and 2. They will be used to subtract the effects of the foreground extinction from the light coming from any other object in the zone under study. 
Table 1. Polarization results

\begin{tabular}{|c|c|c|c|c|c|c|c|c|c|c|c|}
\hline \multirow[b]{2}{*}{ Star $^{a}$} & \multicolumn{5}{|c|}{ Observed } & \multirow[b]{2}{*}{$E_{B-V}$} & \multicolumn{5}{|c|}{ Intracluster } \\
\hline & $\begin{array}{r}\lambda_{\max } \\
\mu \mathrm{m}\end{array}$ & $\epsilon_{\lambda_{\max }}$ & $\begin{array}{c}P_{\max } \mathrm{b} \\
\%\end{array}$ & $\epsilon_{\mathrm{p}}$ & $\sigma_{1}{ }^{\mathrm{c}}$ & & $\begin{array}{r}\lambda_{\max } \\
\mu \mathrm{m}\end{array}$ & $\epsilon_{\lambda_{\max }}$ & $\begin{array}{c}P_{\max } \\
\%\end{array}$ & $\epsilon_{\mathrm{p}}$ & $\sigma_{1}$ \\
\hline 150 & .55 & .10 & 2.85 & .30 & 1.41 & 0.76 & .55 & .29 & 0.99 & 0.31 & 1.69 \\
\hline 166 & .54 & .11 & 4.22 & .56 & 2.15 & 0.88 & .56 & .17 & 2.70 & 0.52 & 2.11 \\
\hline 175 & .40 & .03 & 5.43 & .46 & 0.54 & 1.16 & .42 & .05 & 4.11 & 0.43 & 0.59 \\
\hline 197 & .73 & .04 & 1.82 & .11 & 0.24 & 0.77 & .27 & .03 & 1.41 & 0.25 & 0.21 \\
\hline 205 & .57 & .06 & 2.42 & .16 & 1.30 & 0.79 & .88 & .38 & 1.00 & 0.42 & 1.23 \\
\hline 223 & .59 & .01 & 4.01 & .04 & 1.65 & 0.85 & .59 & .01 & 2.47 & 0.04 & 1.37 \\
\hline 231 & .61 & .01 & 5.31 & .06 & 1.23 & 1.01 & .62 & .02 & 3.55 & 0.05 & 0.88 \\
\hline 235 & .59 & .01 & 5.06 & .09 & 1.02 & 1.15 & .60 & .02 & 3.22 & 0.07 & 0.89 \\
\hline 245 & .30 & .07 & 4.47 & .78 & 0.38 & 0.79 & .24 & .12 & 4.02 & 3.94 & 0.44 \\
\hline 246 & .56 & .03 & 4.53 & .14 & 1.34 & 1.16 & .56 & .03 & 2.80 & 0.11 & 1.07 \\
\hline 251 & .53 & .05 & 5.86 & .37 & 1.65 & 1.00 & .51 & .06 & 4.06 & 0.34 & 1.50 \\
\hline 254 & .53 & .02 & 2.88 & .07 & 1.52 & 0.73 & .50 & .05 & 1.14 & 0.07 & 1.41 \\
\hline 259 & .56 & .03 & 4.70 & .19 & 1.74 & 1.00 & .56 & .07 & 2.91 & 0.22 & 1.94 \\
\hline 275 & .60 & .08 & 2.66 & .16 & 1.87 & 0.72 & .43 & .14 & 1.01 & 0.22 & 2.18 \\
\hline 280 & .51 & .09 & 4.24 & .49 & 0.66 & 0.73 & .56 & .16 & 2.87 & 0.52 & 0.79 \\
\hline 297 & .57 & .05 & 4.01 & .16 & 1.87 & 0.92 & .57 & .08 & 2.20 & 0.16 & 1.86 \\
\hline 301 & .54 & .02 & 2.71 & .07 & 1.41 & 0.95 & .47 & .05 & 0.87 & 0.08 & 1.31 \\
\hline 306 & .50 & .02 & 3.83 & .11 & 0.93 & 0.92 & .44 & .03 & 2.02 & 0.14 & 0.90 \\
\hline 311 & .63 & .04 & 3.08 & .12 & 1.56 & 0.76 & .74 & .16 & 1.48 & 0.24 & 2.00 \\
\hline 313 & .61 & .03 & 2.32 & .05 & 0.81 & 0.72 & 1.24 & .63 & 0.79 & 0.67 & 1.10 \\
\hline 314 & .58 & .00 & 3.43 & .01 & 0.32 & 0.88 & .60 & .01 & 1.57 & 0.01 & 0.26 \\
\hline 343 & .66 & .03 & 3.62 & .09 & 0.65 & 1.11 & .75 & .08 & 1.84 & 0.15 & 0.67 \\
\hline $349 *$ & .63 & .03 & 1.75 & .03 & 1.38 & 0.52 & $\ldots$ & $\ldots$ & $\ldots$ & $\ldots$ & $\ldots$. \\
\hline 351 & .57 & .05 & 3.10 & .20 & 0.67 & 0.71 & .58 & .15 & 1.37 & 0.25 & 0.86 \\
\hline $367 *$ & .60 & .02 & 2.21 & .04 & 0.36 & 0.54 & $\ldots$ & $\ldots$ & $\ldots$ & $\ldots$ & $\ldots$ \\
\hline $374 *$ & .60 & .04 & 2.01 & .06 & 1.47 & 0.56 & $\ldots$ & $\ldots$ & $\ldots$ & $\ldots$ & $\ldots$. \\
\hline 401 & .57 & .02 & 3.10 & .10 & 1.31 & 0.71 & .50 & .05 & 1.55 & 0.12 & 1.53 \\
\hline 406 & .69 & .04 & 2.33 & .08 & 0.51 & 0.88 & .33 & .04 & 1.46 & 0.28 & 0.52 \\
\hline 411 & .59 & .10 & 1.93 & .04 & 1.43 & 0.10 & $\ldots$ & $\ldots$ & $\ldots$ & $\ldots$ & $\ldots$ \\
\hline 444 & .48 & .08 & 3.43 & .36 & 0.67 & 1.06 & .60 & .17 & 1.98 & 0.34 & 0.71 \\
\hline $455 *$ & .50 & .05 & 1.58 & .10 & 1.42 & 0.66 & $\ldots$ & $\ldots$ & $\ldots$ & $\ldots$ & $\ldots$ \\
\hline 503 & .45 & .03 & 0.81 & .07 & 0.58 & 0.80 & .70 & .03 & 1.40 & 0.03 & 0.51 \\
\hline
\end{tabular}

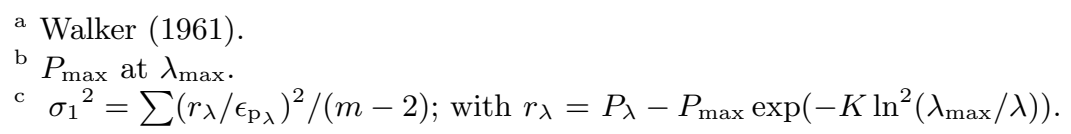

Having in mind that we are handling observed parameters of the dust we proceeded to average them instead of simply obtaining a mean value for each bandpass. Thus the weighted mean of the $\lambda_{\max }$ value for the "frontside" stars is:

$\overline{\lambda_{\max }}=0.58 \pm 0.06 \mu \mathrm{m}$.

For the polarization and orientation of the electric vector $\left(\theta_{V}\right)$ in equatorial coordinates, we get mean values:

$\overline{P_{\max }}=1.88 \pm 0.02 \%$

and

$\overline{\theta_{V}}=75.3 \pm 2.7$

respectively.

Now, through the use of the Serkowski relation (1), we can calculate a distribution $\bar{P}_{\lambda}$ for each bandpass that should characterize the "frontside" stars, in the mean. Then, it is possible to calculate mean Stokes parameters $\overline{Q_{\lambda}}$ and $\overline{U_{\lambda}}$ for the group. Subtracting these mean values from the individuals $Q_{\lambda}$ and $U_{\lambda}$ for the rest of the observed members of NGC 6611 and inverting the procedure, we obtain the values $P_{\text {intracluster }}(\lambda)$ and $\theta_{\text {intracluster }}(\lambda)$, which are listed on the right side of Table 1 .

Once more, the maximum wavelength (in $\mu \mathrm{m}$ ) at which $P_{\max }$ occurs has been calculated by fitting the intracluster polarization in the five bandpasses to the standard Serkowski polarization law for the "non-frontside" stars. The results are presented on the right side of Table 2, together with their correspondent $\lambda_{\max }$ values.

From their intra-cluster polarization values 8 objects (stars Nos. 150, 166, 251, 259, 275, 297, 311 and 401) have 
a value for the intracluster unit weight error of the fit $\left(\sigma_{1}\right)$ above 1.5. Such value may be considered as a limit due to the weighting scheme, that is: a higher value could be indicative of the presence of intrinsic polarization. Stars 175, 275 (recently mentioned), 306 and 406 have their fitted $\lambda_{\max }$ shorter than the average general value for the interstellar medium $(0.55 \mu \mathrm{m})$. This second rejection criterion usually gives another clue to intrinsic polarization. Star 197 stands out as a polarimetric variable as it results from comparing $P_{R}$ observations from Carrasco et al. (1975) and this work, see Fig. 1.

Some objects falling into this two criteria display emission-lines spectra: Nos. 251 and 311 (B2 Ve and B2.5 Ve, respectively; Hillenbrand et al. 1993), Nos. 297 and 306 (B0.5 Ve and B2-B3e, respectively; de Winter et al. 1997), Star 175 has spectral type O5.5 V ((f)) (Hillenbrand et al. 1993). The fitting of the Serkowski's law to star No. 313 gives an abnormal value of $1.24 \mu \mathrm{m}$ on the right side of Table 2 . This star has been mentioned earlier as a visual variable star. The most notable $P_{\lambda}$ and $\theta_{\lambda}$ vs. $\lambda$ plots are shown in Fig. 2, where the solid curve denotes the Serkowski polarization relation for the general interstellar medium. In particular, the plot for star 275 resembles that for star 109 in the polarimetric study of IC 2944 (Vega et al. 1994), and also the stars in Table 4 (Figs. 4 and 5) in Waldhausen \& Marraco (1982), perhaps denoting the presence of circumstellar scattering.

In order to disentangle the relationship between the reddening and the polarization produced by the dust along the line of sight, the upper plot of Fig. 3 examines the relation between $P_{\max }$ and color excess $E_{B-V}$ for the observed stars in NGC 6611. Objects shown as open circles indicate our "frontside" stars. Most objects are located to the right of the interstellar maximum line:

$P_{\max }<3 A_{V} \simeq 3 R_{V} E_{B-V}$

which is derived for the interstellar dust particles (Hiltner 1956). For comparison purposes this relation is also shown in the plot adopting two different values of $R_{V}$ : 3.1 , the "normal" value for the interstellar medium; and 3.75 , the most frequent value in the work of Hillenbrand et al. (1993). The only star located to the left of both relations is No. 411. This object has been classified as a K5 III star by de Winter et al. (1997). It is considered to be a nonmember of NGC 6611, with an $E_{B-V}=0.10 \mathrm{mag}$. Polarimetrically, it shows a $P_{\max }$ value of $1.93 \pm 0.41 \%$ (for $\lambda_{\max }=0.58 \pm 0.03 \mu \mathrm{m}$ ), which is relatively high when related to its excess. Its observed plots have been included in Fig. 2, where an abnormal curve can be seen that reflects more than one origin: interstellar plus circumstellar.

For the interstellar medium, Serkowski et al. (1975) have found a value of 5.03 for the ratio $P_{\max } / E_{B-V}$. In the case of NGC 6611 the stars in the upper plot seem to cluster around 4.2, a value equal to the sample of Carrasco et al. (1975) for NGC 6611. This situation
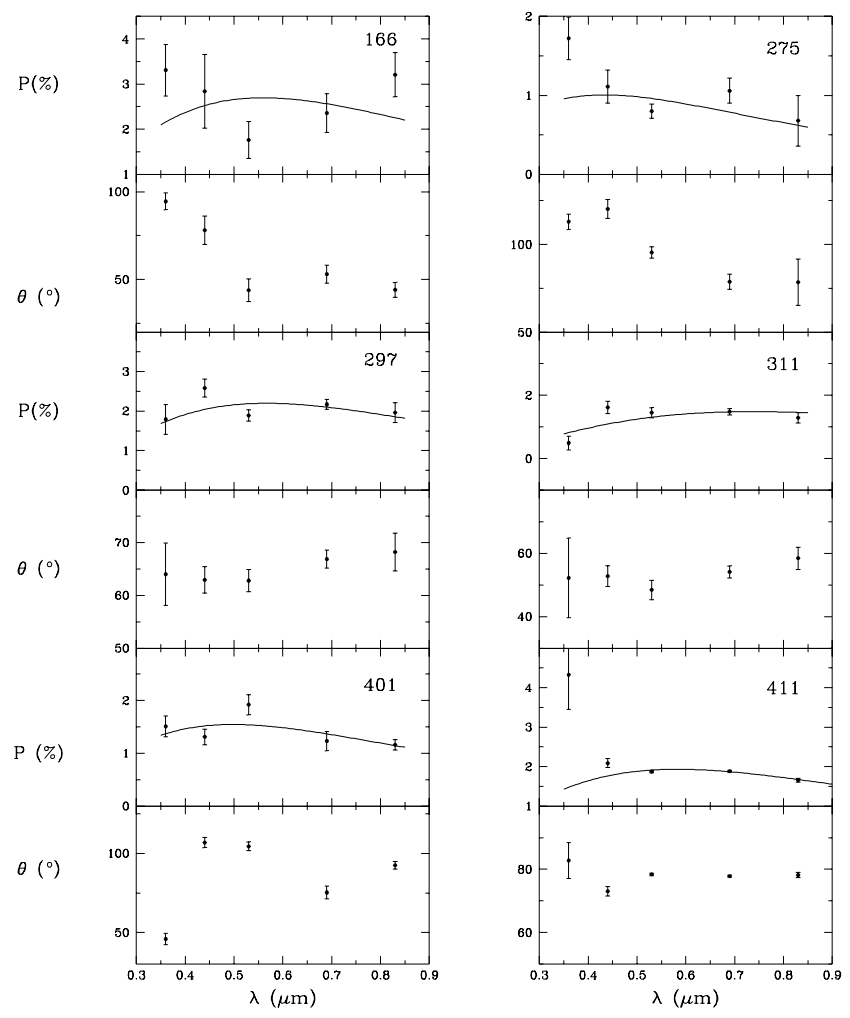

Fig. 2. Intracluster polarization and position angle dependence with wavelength for some of the stars with indications of intrinsic polarization (mismatch between observations and Serkowski's curve fit and/or variable position angle). In the case of star 411 , both plots correspond to observed values

denotes a mean polarization efficiency coming from a combined effect of intracluster and foreground material. Although the observed ratio $P_{\max } / E_{B-V}$ may depend on several elements, mainly on the alignment efficiency, the magnetic field strength and the angle between the magnetic field and the line of sight, in this case it shows the depolarization due to radiation crossing at least two dust clouds with different field directions.

The lower plot in Fig. 3 shows the situation for the same group of stars when we subtract the effects of polarization and reddening due to the material in front of the cluster. Open circles indicate, again, the "frontside" stars. No. 411 has been excluded from this plot and also from the intra-cluster values in Tables 1 and 2 . In this plot, only two stars are located to the left of the maximum line (2): No. 245 is a visual variable, of spectral type B6e and with suspected circumstellar material as noted by de Winter et al. (1997); and No. 280 being a fast rotation variable emission line star (Hillenbrand et al. 1993). From this plot we infer that most of the stars apparently are not strongly affected by intrinsic polarization. The dominant mechanism of polarization in the observed section of NGC 6611 is, therefore, supposed to be the alignment of grains by a magnetic field, in a similar way as that found 


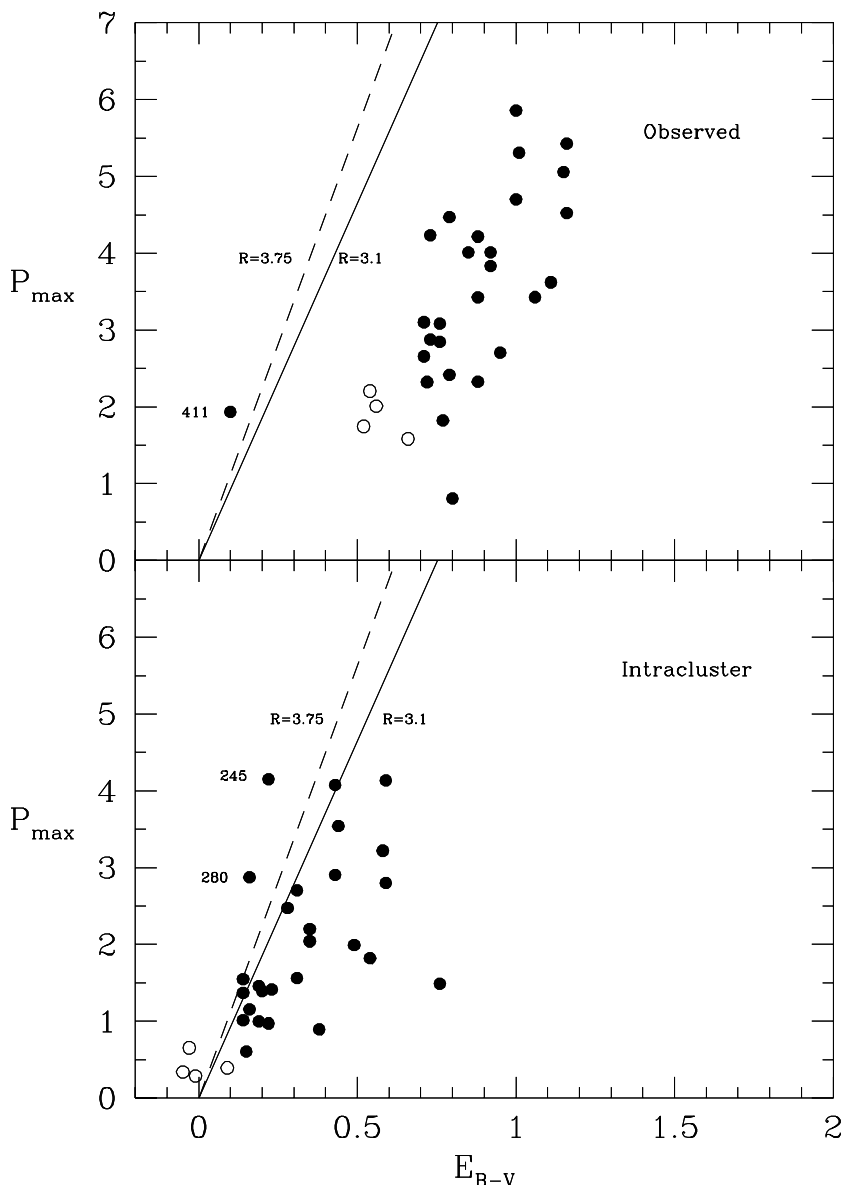

Fig. 3. Polarization efficiency diagrams for observed (upper plot) and intracluster parameters (lower plot). Using $R_{V}=3.1$, the line of maximum efficiency is drawn in both diagrams. The same line but for $R_{V}=3.75$, is also shown. Open circles are used for our "frontside" stars

in the general interstellar medium, with relatively good efficiency.

Figure 4 includes the plot of $P_{\lambda} / P_{\max }$ vs. $\lambda / \lambda_{\max }$ for those stars not excluded from any of the criteria explained in the previous paragraphs showing that their polarization is fully of interstellar origin. Only these stars will be used to test the canonical relations hereinafter. Star 503 was not included because it is a Herbig Ae/Be. It will be mentioned again later.

For member stars in NGC 6611, individual $(V-K)$ values have been taken from the works of Hillenbrand et al. (1993) and Chini \& Wargau (1990); the excesses $E_{V-K}$ were calculated from the relationships (Johnson 1966):

$E_{V-K}=(V-K)-(V-K)_{0}$

where

$(V-K)_{0}=1.05 Q$

and

$Q=(U-B)-\alpha(B-V)$

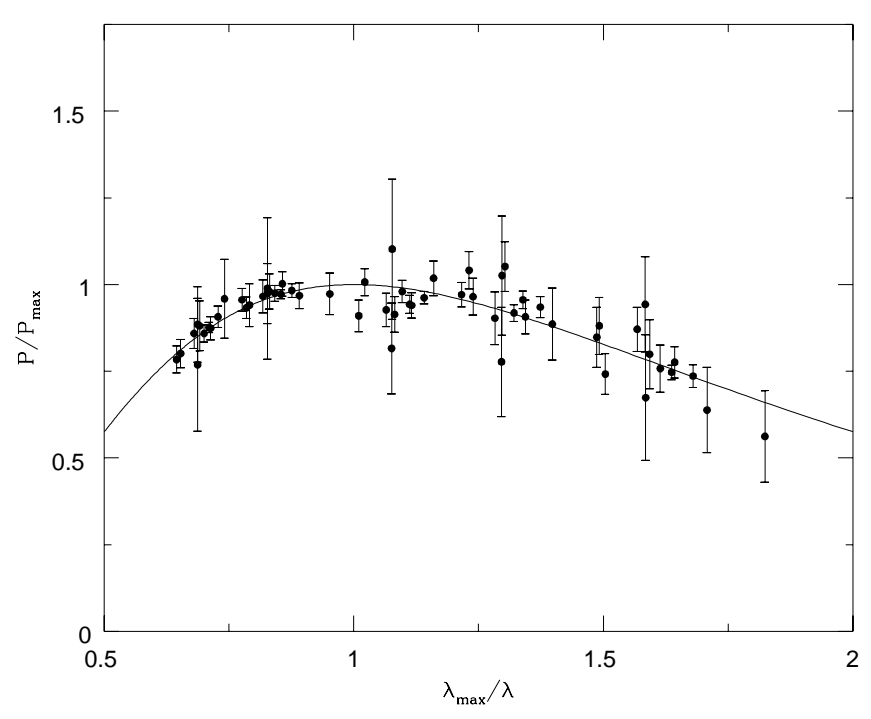

Fig. 4. $P_{\lambda} / P_{\max }$ vs. $\lambda_{\max } / \lambda$ plot for the observed stars not excluded from any of the explained criteria

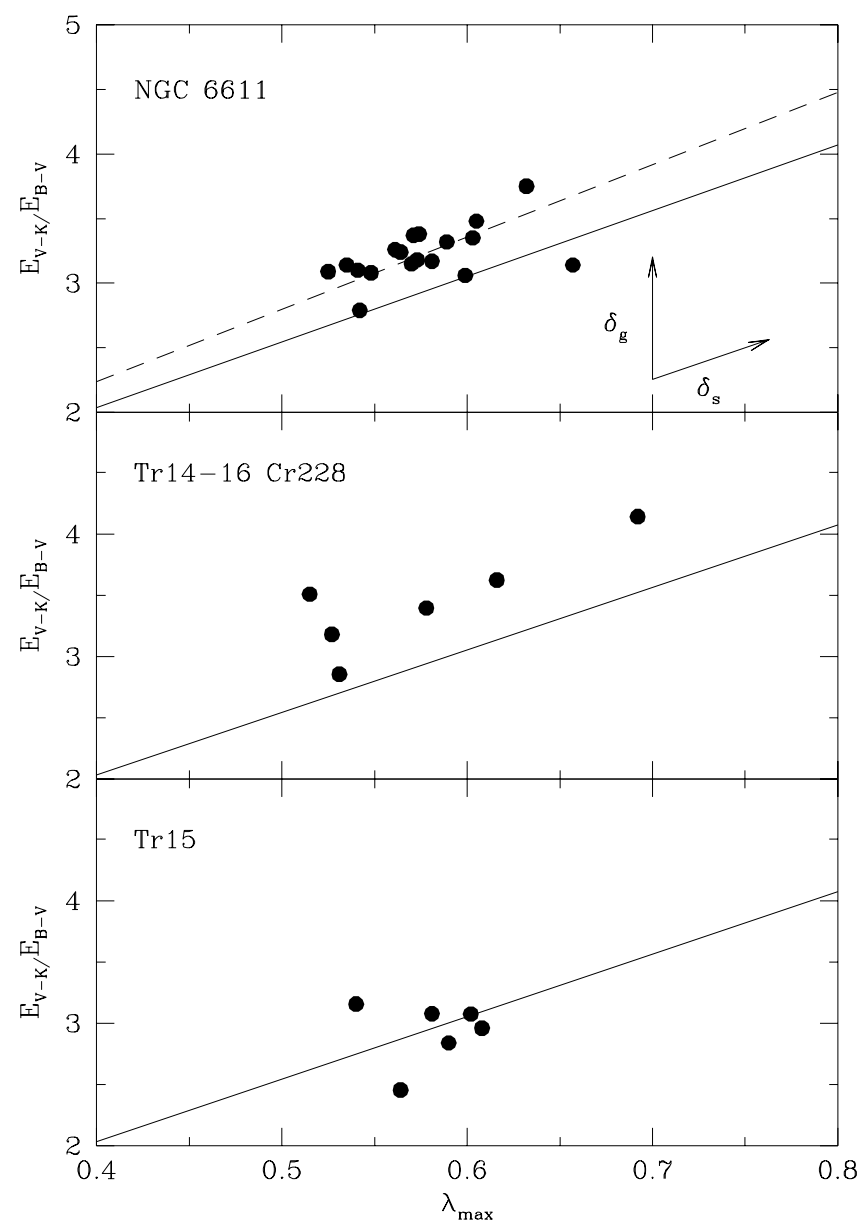

Fig. 5. $E_{V-K} / E_{B-V}$ vs. $\lambda_{\max }$ plots for stars belonging to NGC 6611 (upper), Tr 14/16-Cr 228 (middle) and $\operatorname{Tr} 15$ (lower). In the upper plot, the vectors intend to show the first order results of a mean particle size increase 


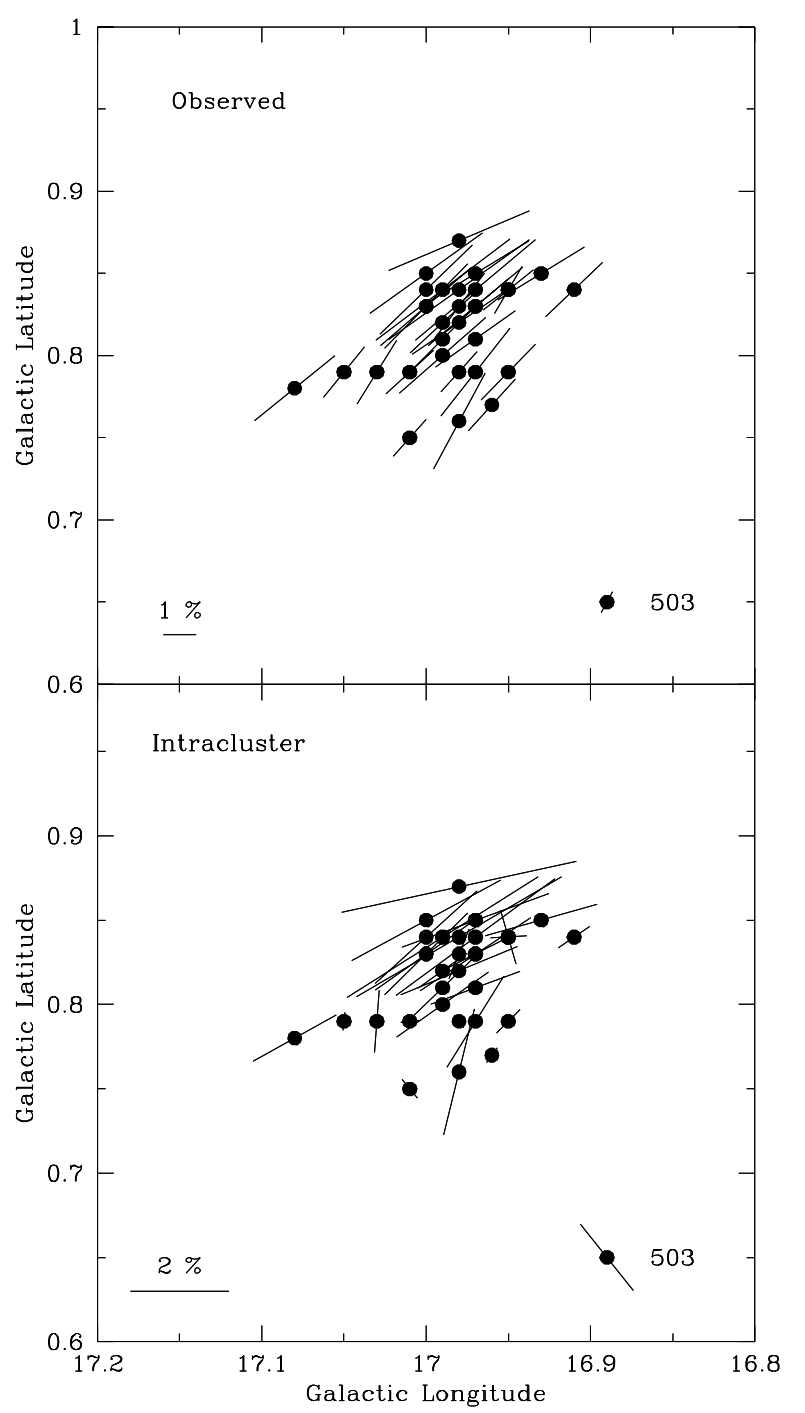

Fig. 6. Observed (upper plot) and intracluster (lower plot) polarization vectors and their orientations for stars belonging to NGC 6611. The length of each vector is proportional to the percentage polarization

where $\alpha$ takes values in the range 0.69 to 0.72 . As for the particular case of NGC 6611, we have adopted $\alpha=0.72$ in the calculations of the $Q$ parameter. This procedure is justified by the fact that it is the same one used by Hillenbrand et al. (1993) and subsequently by Belikov et al. (1999). At the same time, this value is also very similar to the one quoted by Turner (1976) for the cluster, namely $\alpha=0.74$.

In order to check the canonical relationship between $R_{V}$ and $\lambda_{\max }$ for the interstellar dust we have plotted in the upper panel of Fig. 5 only those stars not suspected of having some kind of intrinsic polarization. For comparison purposes we have also plotted stars from the open clusters Tr 14/16 and Cr 228, embedded within the Carina Nebula (an H II region similar to $\mathrm{M} 16$ ), in the middle panel; and from the open cluster $\operatorname{Tr} 15$, to the north of the other two clusters but just outside the $\mathrm{H}$ II region, in the lower panel. The $\lambda_{\max }$ data for these two plots come from Marraco et al. (1993) and the infrared photometry is from Tapia et al. (1988a). The plot for Tr $14 / 16-C r 228$ is identical to the upper panel in Fig. 3 of Marraco et al. (1993). The canonical relation of $E_{V-K} / E_{B-V}=5.09 \lambda_{\max }$ is drawn in the three panels as a solid line.

The dashed line in the upper panel was fitted to the 18 observed stars in NGC 6611 giving $E_{V-K} / E_{B-V}=$ $5.6 \lambda_{\max }$. This means that if we adopt $A_{V} / E_{V-K}=1.12$ we get

$R_{V}=6.3 \lambda_{\max }$

in comparison with a canonical value for the constant in (3) of 5.5.

As previously suggested for Carina by Tapia et al. (1988b) and subsequently confirmed by Marraco et al. (1993), Fig. 5 clearly demonstrates that the canonical relation between $E_{V-K} / E_{B-V}$ and $\lambda_{\max }$ is not valid for stars belonging to dusty $\mathrm{H}$ II regions, as is the case for M 16 and the Carina Nebula, but remains in use for Tr 15 , located well outside the H II region.

Chini \& Wargau (1990) have attempted to model the NGC 6611 dust properties in order to fit the extinction curves obtained with their UBVRIJHKL photometry. Using the MRN graphite-silicate dust model (Mathis et al. 1977; Chini \& Krugel 1983), they obtained a close fit by increasing (in relation with the model representing the standard ISM) the mean graphite grain size by a factor of 2 , while the mean size of silicate grains was increased slightly by a factor of $1.20 \pm 0.09$. As it is widely known (Whittet 1996; Li \& Greenberg 1998), the polarization is accounted for only by silicate grains because graphites are difficult to align. Our mean intracluster $\lambda_{\max }$ is $0.62 \pm 0.10 \mu \mathrm{m}$ and, if we adopt for the ISM a $\overline{\lambda_{\max }}$ value of $0.55 \pm 0.05 \mu \mathrm{m}$, we find that the mean size of the particles responsible for the polarization within the observed region in NGC 6611 is increased by a factor of $1.13 \pm 0.09$ relative to the general ISM, in general agreement with the ideas of Chini \& Wargau (1990). Returning to Fig. 5 we are able to understand now that the canonical relationship between $R_{V}$ and $\lambda_{\max }$ is expected to follow the extinction in the standard ISM where the shape of the extinction curve $\left(R_{V}\right)$ is mainly accounted for the mean silicate grain size. In dusty H II regions, as it is the case of this paper and Tr14/16Cr 228, variations in $R_{V}$ are also due to changes in mean graphite grain size. In both cases, standard ISM and dusty H II regions, $\lambda_{\max }$ varies along with mean silicate grain size. The vectors inserted in the upper panel of Fig. 5 intend to show the first order results of a mean particle size increase. This is the reason why our polarization observations of NGC 6611 and also those of the $\eta$ Carinae nebula do not follow the canonical relationship between $R_{V}$ and $\lambda_{\max }$ of the general interstellar medium. McMillan (1978) concluded that an unimodal size distribution of dielectric 


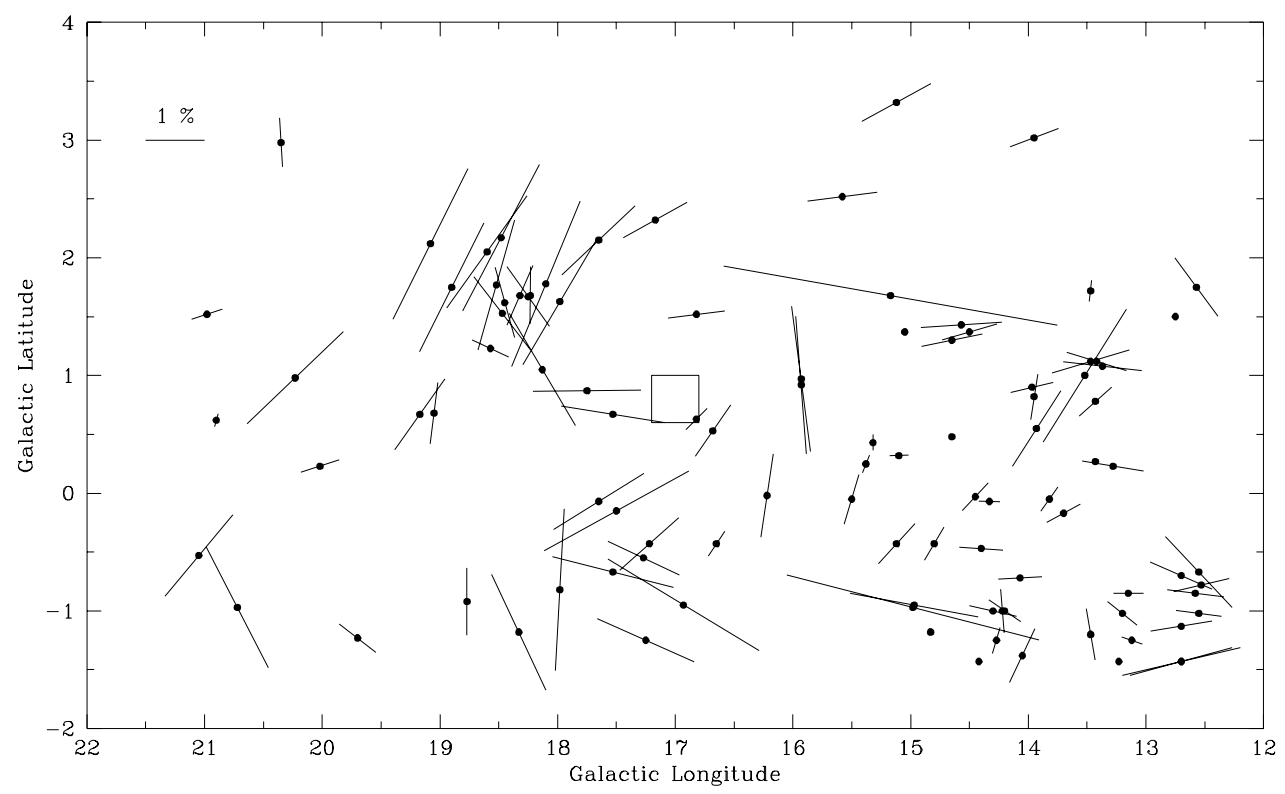

Fig. 7. Polarization vectors and their orientations for stars from the catalog of Axon \& Ellis (1976) in the neighborhood of NGC 6611. The length of each vector is proportional to the percentage polarization. The approximate position of the cluster in the region is indicated with a square whose borders are those of Fig. 6 particles could not account for the position of 3 Orion stars observed by Breger (1977) in the $R_{V}$ vs. $\lambda_{\max }$ diagram. This further points to graphite grain growth as an important factor in changing the shape of the interstellar extinction curve in the direction of dusty $\mathrm{H}$ II regions.

Figure 6 depicts the polarization vectors and their orientations for observed and intra-cluster values (upper and lower plots, respectively).

The foreground polarization has an average direction in galactic coordinates of $131.2 \pm 7.2$ (69.7 in equatorial coordinates), while the intra-arm polarization amounts to $117^{\circ} .1 \pm 18^{\circ} .7$ (55.6 in equatorial coordinates); that is, the projected magnetic field interior to the cluster is somewhat different from the foreground field. As mentioned earlier, star No. 503 with an $\boldsymbol{e}$-vector of $\theta_{V}=157^{\circ} .4$, deviates significantly from the average direction, as other stars from the group with suspected circumstellar dust shells do. Figure 7 shows the observed polarizations in the cluster area from the catalog of Axon \& Ellis (1976). The line of sight to M 16 looks into the Sagittarius arm; in spite of this situation, no alignment of the e-vector of the observed field stars polarization is apparent. To conclude neither the field nor the intracluster polarization are particularly aligned, but the later is closer to the direction of the magnetic field that runs along the arm $\left(\theta_{\mathrm{G}}=90^{\circ}\right)$.

\section{Conclusions}

About $50 \%$ of the observed stars (14 out of 28) in NGC 6611 present indications of measured intrinsic polarization. A similar situation was found in the polarimetric study of IC 2944 (Vega et al. 1994), another open cluster in close relation with an $\mathrm{H}$ II region (RWC62). On the other hand, practically no observed members showed polarization due to a non-interstellar origin in Hogg 15 and Lynga 14 (Orsatti et al. 1998), two open clusters but without any association with gas.

The NGC 6611 intracluster polarization amounts to $\overline{P_{V}}=1.99 \%$ (mean of 14 stars). When compared to the polarization found for the "frontside" stars (1.88\%), we confirm the presence of polarizing dust in association with the observed members of NGC 6611.

The mean intra-cluster $\lambda_{\max }$ value associated with the observed stars gives $\overline{\lambda_{\max }}=0.62 \pm 0.10 \mu \mathrm{m}$ (mean of 14 stars). This dispersion indicates the coexistence of different grain size distributions. When compared to the $\lambda_{\max }$ value for the general interstellar medium, it implies a certain enlargement in mean silicate grain size. Also, it was found that the canonical relation $R_{V}=5.5 \lambda_{\max }$ is not valid for stars in dusty $\mathrm{H}$ II regions like the observed portion of M 16 and the Carina Nebula where it was previously shown by Tapia et al. (1988b) and subsequently confirmed by Marraco et al. (1993). This may arise mainly from the presence of these before mentioned silicate grains of slightly larger size than the standard ISM and also from a considerable increase in mean graphite grain size, in accordance with previous results from Chini \& Wargau (1990).

We plan to extend the observations to other parts of the $\mathrm{H}$ II region less connected with dense dust clouds to help clarify the validity of this non canonical relation and to re-observe for variability some stars of the present paper in order to further explain the circumstellar polarization in more detail.

Acknowledgements. We wish to acknowledge the technical support and hospitality at CASLEO during the observing runs and also the support of the Facultad de Ciencias Astronómicas y 
Geofísicas de La Plata (UNLP). Special thanks go to Mrs. M. C. Fanjul de Correbo and to Mr. Ruben E. Martínez for technical assistance. An anonymous referee, who pointed out several key items and generously improved the manuscript, is also thanked.

\section{References}

Axon D.J., Ellis R.S., 1976, MNRAS 177, 499

Belikov A.N., Kharchenko N.V., Piskunov A.E., Schilbach E., 1999, A\&AS 134, 525

Breger M., 1977, ApJ 215, 119

Carrasco L., Strom K.M., Strom S.E., 1975, Rev. Mex. Astron. Astrofis. 1, 283

Chini R., Krugel E., 1983, A\&A 117, 289

Chini R., Wargau W.F., 1990, A\&A 227, 213

Clocchiatti A., Marraco H.G., 1988, A\&A 197, L1

Feinstein A., Marraco H.G., 1971, PASP 83, 218

Gebel W.L., 1968, ApJ 153, 743

Hillenbrand L.A., Massey P., Strom S.E., Merrill K.M., 1993, AJ 106, 1906

Hiltner W.A., 1956, ApJS 2, 389

Hoag A.A., Johnson H.L., Iriarte B., et al., 1961, Publ. U.S. Naval Obs. Second Serie 17, 345

Johnson H.L., 1966, ARA\&A 4, 193

Johnson H.L., 1968, in Nebulae and Interstellar Matter, Stars and Stellar Systems VII, Middlehurst B.M. and Aller L.H. (eds.), p. 167

Li A., Greenberg J.M., 1998, A\&AS 591

Marraco H.G., Vega E.I., Vrba F.J., 1993, AJ 105, 258
Mathis J.S., Rumpl W., Nordsiek K.H., 1977, ApJ 217, 425

McMillan R.S., 1978, ApJ 225, 880

Orsatti A.M., Vega E.I., Marraco H.G., 1998, AJ 116, 266

Sagar R., Joshi U.C., 1979, Ap\&SS 66, 3

Serkowski K., 1973, in IAU Symp. 52, Interstellar Dust and Related Topics, Greenberg J.M. and van der Hulst H.C. (eds.). Dordrecht: Reidel, p. 145

Serkowski K., Mathewson D.S., Ford V.Ł., 1975, ApJ 196, 261

Tapia M., Roth M., Marraco H.G., Ruiz M.T., 1988a, MNRAS 232,661

Tapia M., Roth M., Marraco H.G., Ruiz M.T., 1988b, in Conference on Dust in the Universe, Bailey M.E. and Williams D.A. (eds.). Cambridge University Press, Cambridge, p. 19

Thé P.S., de Winter D., Feinstein A., Westerlund B.E., 1990, A\&AS 82, 319

Turner D.G., 1974, unpublished Ph. D. Thesis, University of Western Ontario

Turner D.G., 1976, AJ 81, 1125

Turner D.G., 1994, Rev. Mex. Astron. Astrofis. 29, 163

Vega E.I., Orsatti A.M., Marraco H.G., 1994, AJ 108, 1834

Waldhausen S., Marraco H.G., 1982, AJ 87, 1730

Walker M.E., 1961, ApJ 133, 438

Whittet D.C.B., 1996, in Polarimetry of the Interstellar Medium, ASP Conf. Ser. 97, Roberge W.G. and Whittet D.C.B. (eds.) p. 125

Whittet D.C.B., van Breda I.G., 1978, A\&A 66, 57

Wilking B.A., Lebofsky M.J., Martin P.G., Rieke G.H., Kemp J.C., 1980, ApJ 235, 905

de Winter D., Koulis C., Thé P.S., et al., 1997, A\&AS 121, 223 